\title{
Comparison of the brain activations of experienced and novice designers engaged in initiating imagination
}

\author{
Chaoyun Liang ${ }^{*}$; Yu-Cheng Liu ${ }^{2}$ \\ ${ }^{1}$ Professor, Department of Bio-Industry Communication and Development, National Taiwan University, Taipei, Taiwan \\ ${ }^{2}$ Postdoctoral Researcher, Department of Bio-Industry Communication and Development, National Taiwan University, Taipei, \\ Taiwan
}

\author{
*Corresponding Author(s): Chaoyun Liang \\ Professor, Department of Bio-Industry Communica- \\ tion and Development, National Taiwan University, \\ No. 1, Sec. 4, Roosevelt Road, Taipei, 10617, Taiwan \\ Email: cliang@ntu.edu.tw
}

Received: Aug 20, 2018

Accepted: Nov 08, 2018

Published Online: Nov 12, 2018

Journal: Journal of Psychiatry and Behavioral Sciences

Publisher: MedDocs Publishers LLC

Online edition: http://meddocsonline.org/

Copyright: (C) Liang C (2018). This Article is distributed under the terms of Creative Commons Attribution 4.0 International License

Keywords: Brain activation; Electroencephalography; Experienced designer; Initiating imagination; Novice designer

\section{Introduction}

Designers often engage in activities involving the visualization of ideas, a process which primarily relies on their imaginations. Highly imaginative designers can create new forms of meaning and conjure new realities [1,2]. This capacity, known as initiating imagination, is the capability to explore the unknown and productively initiate novel ideas [3]. Initiating imagination is evaluated using two indicators, namely novelty and productivity. Novelty refers to an individual's ability to create uncommon ideas and productivity refers to an individual's ability to productively generate ideas. In addition, Mayseless, et al. [4] indicated

\begin{abstract}
Designers often engage in imaginative activities and their performance largely reflects their unique distinct experiences. Few studies have examined the relationship between creative imagination and designer experience. Thirty visual designers were invited to participate in this study, which examined the differences in the brain activities of Experienced Designers (EDs) and Novice Designers (NDs) engaged in initiating imagination stimulated by abstract images. The following results were obtained. (i) The prefrontal and bilateral frontotemporal brain regions were activated during the idea generation task for the novelty indicator. (ii) The right frontal and temporal regions were activated during the idea evaluation task for the novelty indicator. (iii) During the idea generation task for the productivity indicator, noticeable activations in the right-biased middle frontoparietal region was observed. (iv) During the idea evaluation task for the productivity indicator, noticeable activations in the prefrontal and frontal regions were detected.
\end{abstract}

that the process of producing creative ideas can be divided into two phases: idea generation and idea evaluation. However, few studies have investigated the relationships between designer creativity and their brain activities, as well as the differences between idea generation and idea evaluation [5].

The various working styles of designers reflect their distinct experiences, which are a critical factor influencing design performance [6] and play a decisive role in design thinking as the central inspiration for creating any design object [7]. The topics of designer experience and EExperienced Designers (EDs)

Cite this article: Liang C, Liu YC. Comparison of the brain activations of experienced and novice designers engaged in initiating imagination. J Psychiatry Behav Sci. 2018; 3: 1015. 
have been discussed extensively over the past 30 years. Prior experience enables designers to adopt a conjectural approach to their designs and frame design problems in terms of relevant solutions [8]. EDs are solution focused, and thus they can shift quickly between problem scoping and solution proposing [9]. This coevolution of problems and solutions for EDs is a situated process that depends on specific problem domains and environments [9-11].

EDs can store and access information in larger cognitive chunks and demonstrate more schema-driven analogising than can Novice Designers (NDs) $[9,24]$. EDs can concurrently enact numerous cognitive activities because their knowledge is well organised, which enables them to access and process information more efficiently than can novices [13]. Moreover, most expert designers adhere to a few possible solutions that emerge through the gradual transformation of some core ideas and are eventually proven to be the most effective strategies [14]. The aforementioned studies have focused on the systematic investigation of designer experiences and practices. However, few studies have been conducted regarding the relationship between designer experience and creative thinking. This is particularly notable as regards empirical research involving alternative methodologies such as neuroscience, which has high potential for experimentally tracing the interrelations between design cognition and brain activity $[5,11]$.

In one of the few studies connecting designer experience to neuroscience, Goldschmidt, [15] emphasised that when EDs focus and defocus attention to stimuli, the structure and activity of their neural networks operate in conjunction with their sensitivity, expertise, and flexibility to enable creative idea generation. Specifically, previous studies have identified that the medial temporal lobe stores memories and associations from prior experiences and the medial prefrontal lobe facilitates the flexible use of these memories. These two lobes converge on major integration nodes [16]. Prior research has identified that the Default Mode Network (DMN) is active when people are engaged in internally focused tasks such as retrieving an autobiographical memory, envisioning the future, or considering the perspectives of others $[16,17]$. In addition, robust evidence indicates that the alpha effect is observable during the generation of creative ideas and subjective experiences of emerging insights $[18,19]$.

Liang et al. [11] observed that the visual attention of expert designers takes the form of frontoparietal engagement to select from competing visual stimuli. The left dorsal stream and right ventral stream appeared to be particularly active when the designers were engaged in visual attention tasks. In addition, during a visual association task, the alpha waves of the expert designers were activated in the central region where the Dorsolateral Prefrontal Cortex (DLPFC), Anterior Cingulate Cortex (ACC), and DMN are located, whereas the $\gamma$ waves were activated in the distributed DLPFC, Posterior Cingulate Cortex (PCC), DMN, and V1 regions. Yao et al. [5] further revealed strong connectivity between prior memories and high-order cognitive processing during visual association. Expert designers are more competent in controlling their executive functions to process conflicting concepts, determining future actions in accordance with their goals, and maintaining a balance between aesthetics and rationality than are NDs.

The most effective stimuli for enhancing design creativity are visual, particularly abstract representations, which are distinct from habits of viewing and transcend the sensory experiences accessible in everyday life. Thus, designers' capacity to engage in the creative process relies on high-level visual literacy $[15,20]$. In the current study, to address the lack of scientific evidence regarding the relationship between professional experience and designer imagination, differences in the brain activities of EDs and NDs engaging in initiating imagination stimulated by abstract images were analysed. The specific research questions were as follows:

(i) Which brain regions are particularly activated when EDs and NDs engage in tasks that involve initiating imagination?

(ii) What are the differences in brain activities between EDs and NDs in terms of the two indicators of initiating imagination (i.e., novelty and productivity)?

(iii) What are the differences in brain activities between EDs and NDs in terms of two idea-processing tasks (i.e., idea generation and idea evaluation)?

\section{Methods and materials}

\section{Participants}

In this study, 15 EDs and 15 student visual designers were invited to participate in an Electroencephalography (EEG) experiment. The inclusion criteria for EDs included (i) design-related work experience of more than 15 years, (ii) experience in leading design teams specialising in visual design, and (iii) willingness to participate in the experiment. The inclusion criteria for student designers included (i) being a junior or senior majoring in design, (ii) being recommended by course instructors, and (iii) willingness to participate in the experiment. These criteria allowed for a degree of diversity in both groups to enable the exploration of two distinct ranges of designer experience. All the participants had no history of cardiovascular disorders or drug or alcohol abuse and had normal or corrected-to-normal vision. Because of incomplete participation and signal malfunction, the EEG data of 11 EDs (four women and seven men; age: 31-39 years) and 13 student designers (eight women and five men; age: 21-23 years) were collected for further analysis.

\section{Equipment and materials}

The EEG headset used in this study was a 32-channel inflatable system designed by Brain Rhythm Inc., Taiwan. The headset has two dry foam-based EEG sensors that are used only for the forehead Fp1 and Fp2 sites in the international 10-20 system. The system has 16-bit quantisation and a sampling rate of 250 $\mathrm{Hz}$. It features spring-loaded dry electrodes and a soft cap that can be used repeatedly on hairy sites without conductive gel. EEG data were wirelessly received through a Bluetooth protocol without the need for external cabling.

The visual stimuli used in this study were 10 presentative paintings by Pablo Picasso, probably the most dominant and influential artist of the first half of the twentieth century. Picasso often interchangeably used different styles such as symbolism, abstraction, and surrealism. These stimuli were selected for three reasons: (i) Robust evidence has determined that pictorial representations exert a marked influence on design thinking $[20,21]$; (ii) prior research has indicated that abstract sources with no defined form provide designers with freedom to explore and imagine [20,22]; and (iii) most of Picasso's works were published prior to 1960 and are therefore classified as public domain works in most parts of the world. The usage of these artworks was restricted to use for the purpose of academic re- 
search in this study. The usage of these artworks was restricted for the purpose of academic research in this study. Some of the visual stimuli are displayed in the Appendix.

\section{Experimental procedure}

After signed consent forms were obtained, the participants were invited to sit in comfortable chairs and EEG electrode caps were placed on their heads. They were asked to minimise head and body movement and their baseline (resting state) brain responses were recorded. They were then instructed to spend approximately 2 minutes verbalising the design problem, purpose, and desired outcomes of an ongoing design project. The 10 paintings with questions corresponding to the indicators of initiating imagination (i.e., novelty and productivity) were randomly displayed to the participants in the form of a prerecorded presentation on a 46-inch TV monitor.

Regarding the novelty indicator, each of the paintings was presented for 30 seconds. During the first 15 seconds, each participant was requested to silently respond to the following question: 'What innovative ideas does this painting stimulate and inspire for the project you just mentioned?' EEG data were subsequently recorded. During the second 15 seconds, each participant responded to the question, 'How could these ideas improve the originally imagined outcome?' The first question was related to idea generation, whereas the second was related to idea evaluation. Idea generation involves cycles of idea retrieval and association through memory, whereas idea evaluation involves analysing usefulness and value [4]. Subsequently, the participants verbalised their answers for 2.5 minutes. All answers were tape recorded to assist the researchers in understanding them.

Regarding the productivity indicator, the participants were stimulated by the same set of paintings and asked the following questions: 'What analogues does this painting stimulate and inspire for the project you just mentioned?' and 'How could these analogues improve the originally imagined outcome?' The procedure for this session was the same as that for the previous session. Each painting experiment lasted 3 minutes, and thus the entire EEG measurement lasted 60 minutes. In total, the experiment was completed in approximately 90 minutes including the time required to explain the experiment, test the EEG headset, and describe the project. The experimental process was identical for all participants to ensure enquiry quality. The verbalisation session was aimed at acquiring qualitative data and treated as an intertrial interval to avoid recording overlapping brain responses. The qualitative data were collected for validity triangulation rather than scientific comparison between brainwave activations and narrative contents.

\section{Data analyses}

The EEG data were inspected to remove ill-functioning channels and first divided into 1.6-second signals. Low- and high-pass filters with cutoff frequencies of 50 and $1 \mathrm{~Hz}$, respectively, were applied to all signals based on finite impulse response filters to remove the line noise and direct-current drift [23]. Bad channels were removed by kurtosis in EEGlab. We used 5 standard deviations from the mean as thresholds. Some epochs were excluded manually due to electrical artifacts. The EEGLAB toolbox was used to decompose the filtered EEG signals through Independent Component Analysis (ICA) with the Infomax algorithm, where all components were grouped into several clusters based on outcome similarity.
The signals of each IC were gained from the inner product of the EEG signals and ICA weighting matrix. Nonartefact independent component scalp topographies often strongly resemble the projection of a single dipole and all ICA components were grouped into several clusters according to outcome similarity by using the k-means clustering method [24]. The scalp topography of each independent component was used to plot the Three-Dimensional (3D) location of an equivalent dipole through the DIPFIT plug-in. Each dipole represents the location of an independent component, as well as a specific cortex region. A threshold of residual variance from the scalp projections of these dipoles was set as $15 \%$.

Time-invariant correlations between clusters (cortex regions) were obtained by averaging the dipole signals of each cluster with correlation coefficients. Time-domain data were then transformed into frequency-domain data by using the fast Fourier transform function. The frequency-domain data were defined based on prior research [25-27]. The spectra of the EEG signals were separated into five frequency bands, namely delta (0.5-3.5 Hz), theta $(3.5-8 \mathrm{~Hz})$, alpha (range: 8-13 Hz; low: 8-9 $\mathrm{Hz}$; middle: 9-11 Hz; high: 11-13 Hz), beta (range: $13-30 \mathrm{~Hz}$; low: 13-16 Hz; middle: 16-20 Hz; high: 20-30 Hz), and gamma (range: 30-100 Hz; low: 30-60 Hz; typical: $40 \mathrm{~Hz}$; high: $>60 \mathrm{~Hz}$ ). Only low gamma bands ranging between 30 and $60 \mathrm{~Hz}$ were investigated in the present study because high gamma bands rarely appear in EEG results. A paired-sample Wilcoxon signedrank test was performed to assess the differences in the brain activity spectra of the EDs and NDs.

\section{Results}

Regarding the novelty indicator, both the EDs and NDs exhibited relatively high brain activation in the prefrontal and bilateral frontotemporal regions when engaged in the idea generation task (Figure 1). The colours of the scalp topographies indicate brain activity, with warm colours signifying higher activation and cold colours signifying lower activation. Figures $1 a-1 d$ display the scalp topographies and 3D dipole plots and divide the brain activations into four major component clusters, namely the right prefrontal, left temporal, right temporal, and left frontal cortices. The correlations among these major component clusters are listed in Table 1. Positive associations were observed between the right prefrontal and left temporal cortices and between the right temporal and left frontal cortices.

The differences in spectral power between the EDs and NDs were clarified using the results of the Wilcoxon signed-rank test. The significance levels of the null hypothesis at different frequencies are shown as red dots in the spectra plots. As depicted in Figure 2a (right prefrontal cluster), no significant power difference was observed. In the left temporal cluster, only one significant power difference (EDs > NDs) was observed in the beta band at $21 \mathrm{~Hz}$ ( $p=0.033$; Figure $2 b$ ). The results also indicated that two significant power differences (EDs > NDs) in the right temporal cluster were observed in the gamma band at $37 \mathrm{~Hz}(\mathrm{p}$ $=0.030)$ and the beta band at $18 \mathrm{~Hz}(p=0.044)$ (Figure $2 c)$. Finally, in the left frontal cluster, two significant power differences (NDs > EDs) were observed in the beta band at $27 \mathrm{~Hz}(p=0.033)$ and the gamma band at $41 \mathrm{~Hz}(p=0.038)$ (Figure $2 d)$. 


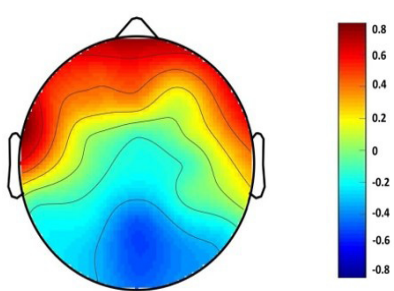

1. Root cluster
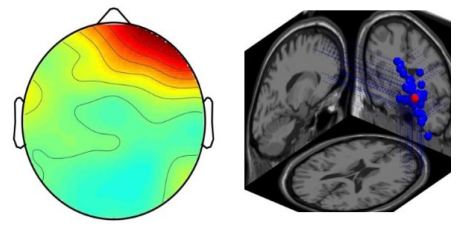

1a. Right prefrontal cluster

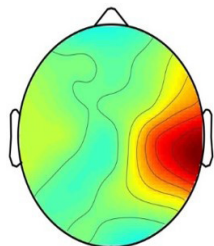

1c. Right temporal cluster

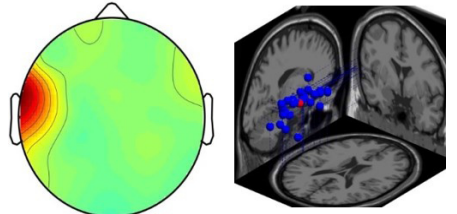

1b. Left temporal cluster

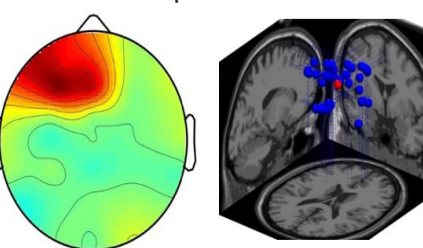

1d. Left frontal cluster
Figure 1: Scalp topographies and 3D dipole plots for the novelty indicator during the idea generation task (1: scalp map for the root cluster; 1a-1d: scalp topographies for the component clusters and their 3D dipole source locations)

Table 1: Correlations among the major components of the novelty indicator in the idea generation task.

\begin{tabular}{|l|c|c|c|c|}
\hline \multicolumn{1}{|c|}{ Component } & $\mathrm{rPF}$ & $\mathrm{IT}$ & $\mathrm{rT}$ & $\mathrm{IF}$ \\
\hline Right prefrontal (rPF) & 1 & $.38^{* * *}$ & $-.13^{*}$ & .00 \\
\hline Left temporal (IT) & & 1 & .00 & -.02 \\
\hline Right temporal (rT) & & & 1 & $.34^{* * *}$ \\
\hline Left frontal (IF) & & & & 1 \\
\hline
\end{tabular}

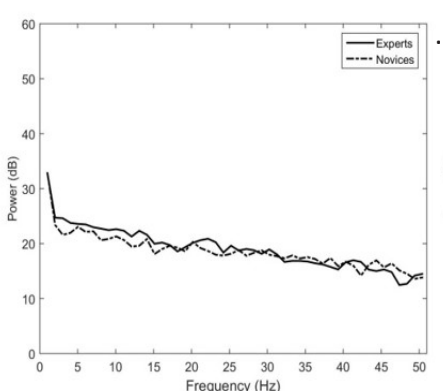

2a. Right prefrontal cluster

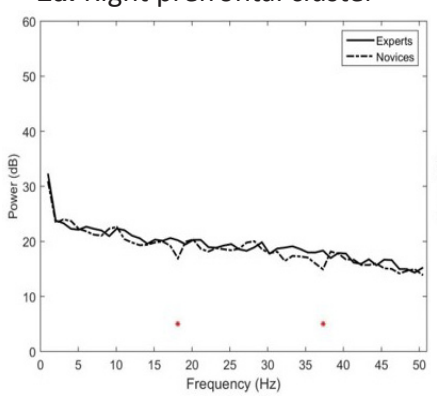

2c. Right temporal cluster

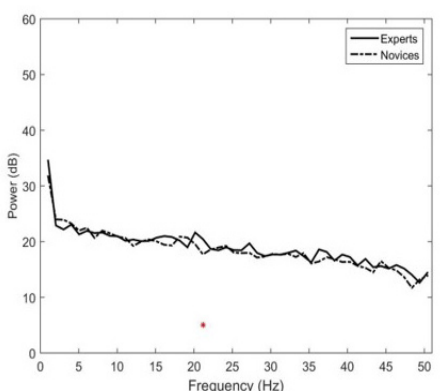

2b. Left temporal cluster

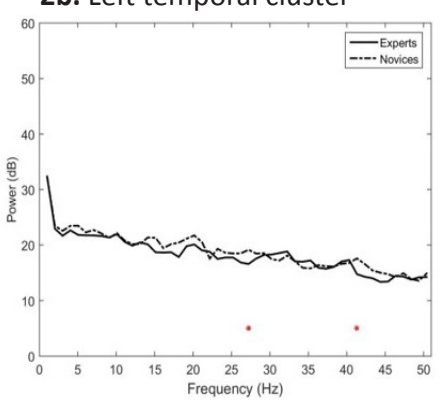

2d. Left frontal cluster
Figure 2: Spectral power and Wilcoxon signed-rank test results for the novelty indicator during the idea generation task

Both the EDs and NDs exhibited high brain activation in the right frontal and temporal cortices when engaged in the idea evaluation task for the novelty indicator (Figure 2). The scalp topographies and 3D dipole plots (Figures 3a-3d) reveal that these brain activations can be separated into four major component clusters: the left temporal, right-biased middle frontal, right lateral frontal, and right temporal cortices. The right-biased middle frontal region is closely associated with the ACC. Table 2 lists the correlations among these major component clusters. The association between the right-biased middle frontal and right temporal cortices was noticeable and positive, whereas that between the right lateral frontal and right temporal cortices was markedly negative.

As depicted in Figure 4a (left temporal cluster), significant power differences (NDs > EDs) appeared in the distributed frequencies. The largest differences appeared in the beta band at $29 \mathrm{~Hz}(p=0.006)$ and $15 \mathrm{~Hz}(p=0.009)$. Significant power differences (NDs > EDs) were also observed in the distributed frequencies in the right-biased middle frontal cluster (Figure 4b), where the largest differences appeared in the gamma band at $43 \mathrm{~Hz}(p=0.017)$ and the beta band at $15 \mathrm{~Hz}(p=0.026)$. Additionally, in the right lateral frontal cluster, only one significant power difference (EDs > NDs) was observed in the beta band at $15 \mathrm{~Hz}$ ( $p=0.022$; Figure $4 \mathrm{c}$ ). Finally, significant power differences were observed in the distributed frequencies in the right temporal cluster (Figure 4d), where the largest differences (NDs $>$ EDs) appeared in the delta band at $2 \mathrm{~Hz}(p=0.004)$ and the gamma band at $45 \mathrm{~Hz}(p=0.038)$.
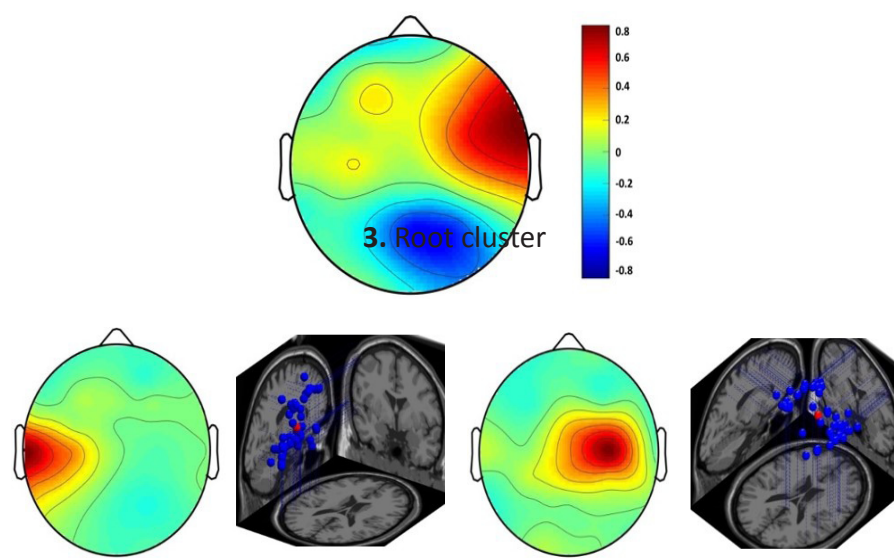

3a. Left temporal cluster 3 b. Right-biased middle frontal cluster

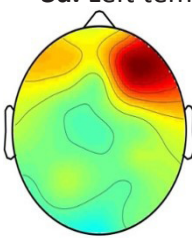

3c. Right lateral frontal cluster
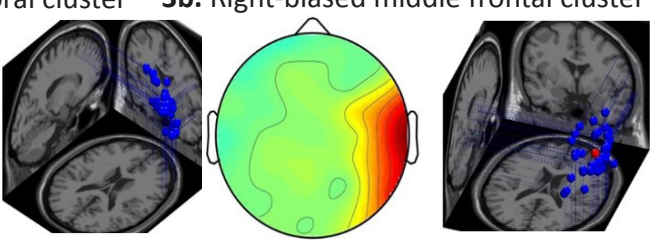

3d. Right temporal cluster

Figure 3: Scalp topographies and 3D dipole plots for the novelty indicator during the idea evaluation task

Table 2: Correlations among the major components of the novelty indicator in the idea evaluation task

\begin{tabular}{|l|c|c|c|c|}
\hline Component & $\mathrm{IT}$ & $\mathrm{rMF}$ & $\mathrm{rF}$ & $\mathrm{rT}$ \\
\hline Left temporal (IT) & 1 & $-.21 * * *$ & $.29 * * *$ & $-.28 * * *$ \\
\hline Right-biased middle frontal (rMF) & & 1 & $-.17^{* * *}$ & $.39 * * *$ \\
\hline Right lateral frontal (rF) & & & 1 & $-.36 * * *$ \\
\hline Right temporal (rT) & & & & 1 \\
\hline
\end{tabular}




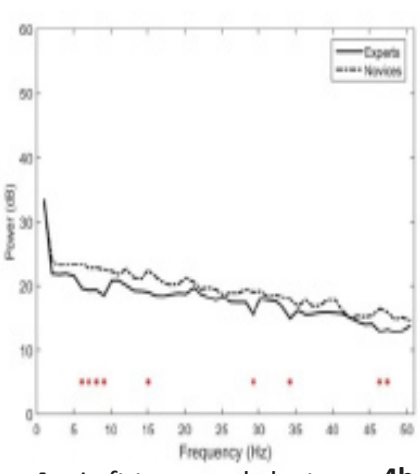

4a. Left temporal cluster

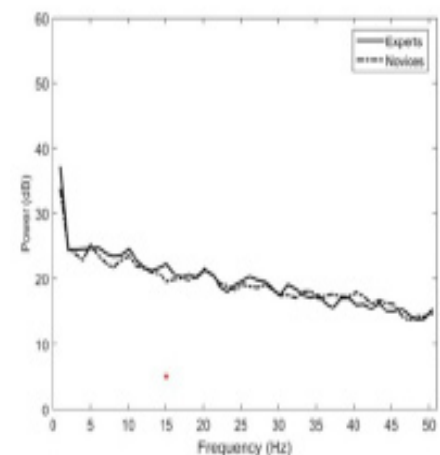

4c. Right lateral frontal cluster

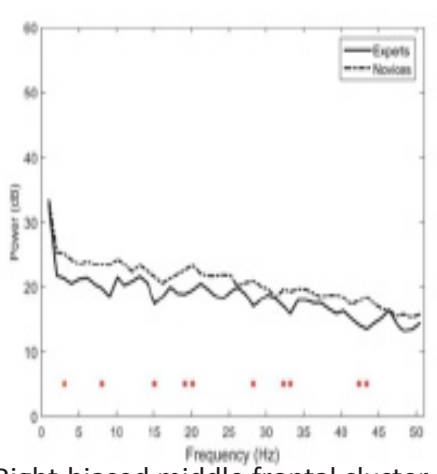

b. Right-biased middle frontal cluster

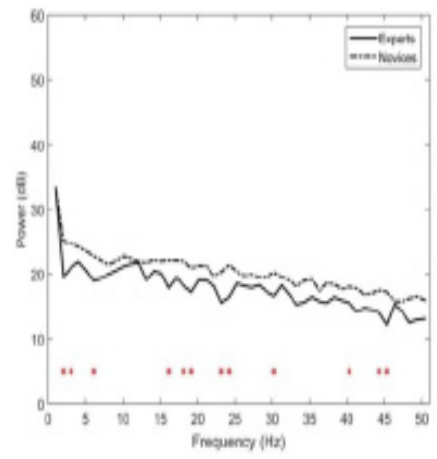

4d. Right temporal cluster

Figure 4: Spectral power and Wilcoxon signed-rank test results for the novelty indicator during the idea evaluation task

Regarding the productivity indicator, the participants exhibited relatively high brain activation in the middle frontal cortex when engaged in the idea generation task (Figure 5). The scalp topographies and 3D dipole plots displayed in Figures $5 a-5 d$ reveal that these brain activations can be separated into four major component clusters, namely the right medial parietal, middle frontal, right temporal, and left lateral parietal cortices. The right medial parietal region is located in the PCC, whereas the middle frontal region is situated in the ACC. The correlations among these major component clusters are listed in Table 3. Relatively high levels of association were observed between the right medial parietal and middle frontal cortices, right medial parietal and left lateral parietal cortices, and middle frontal and left lateral parietal cortices.

In the right medial parietal cluster (Figure 6a), some significant power differences were observed. The largest differences (EDs $>$ NDs) appeared in the alpha band at $8 \mathrm{~Hz}(p=0.005)$ and $10 \mathrm{~Hz}(p=0.009)$. As depicted in Figure $6 b$, in the middle frontal cluster, no significant power differences were observed. In the right temporal cluster (Figure 6c), significant power differences were observed at frequencies of $36 \mathrm{~Hz}$ or higher. The largest differences (NDs > EDs) appeared in the gamma band at $38 \mathrm{~Hz}$ ( $p$ $=0.008)$ and $36 \mathrm{~Hz}(p=0.024)$. Finally, in the left lateral parietal cluster (Figure 6d), most significant power differences appeared at frequencies lower than $16 \mathrm{~Hz}$. The largest differences (EDs > NDs) were observed in the delta band at $1 \mathrm{~Hz}(p=0.007)$ and the theta band at $7 \mathrm{~Hz}(\mathrm{p}=0.007)$.

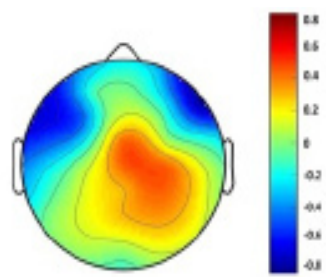

5. Root cluster

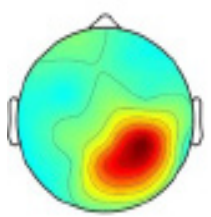

5a. Right medial parietal cluster

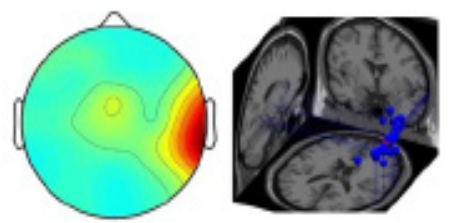

5c. Right temporal cluster

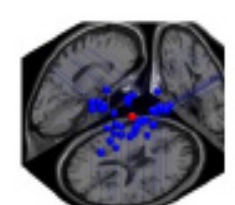

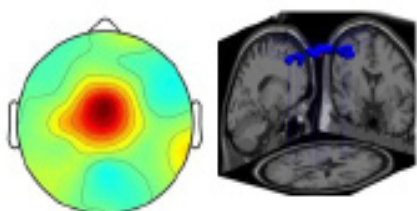

5b. Middle frontal cluster

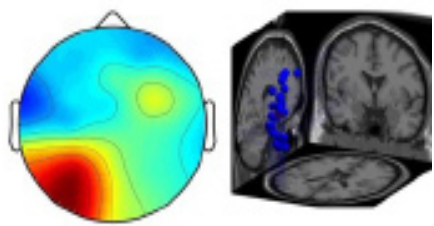

5d. Left lateral parietal cluster
Figure 5: Scalp topographies and 3D dipole plots for the productivity indicator during the idea generation task

Table 3: Correlations among the major components of the productivity indicator in the idea generation task

\begin{tabular}{|l|l|l|l|l|}
\hline Component & $\mathrm{rmP}$ & $\mathrm{mF}$ & $\mathrm{rT}$ & IIP \\
\hline Right medial parietal (rmP) & 1 & $.94 * * *$ & $-.25 * * *$ & $.64 * * *$ \\
\hline Middle frontal (mF) & & 1 & $-.24 * * *$ & $.54 * * *$ \\
\hline Right temporal (rT) & & 1 & $-.35^{* * *}$ \\
\hline Left lateral parietal (IIP) & & & & \\
\hline
\end{tabular}

6a. Right medial parietal cluster

6b. Middle frontal cluster

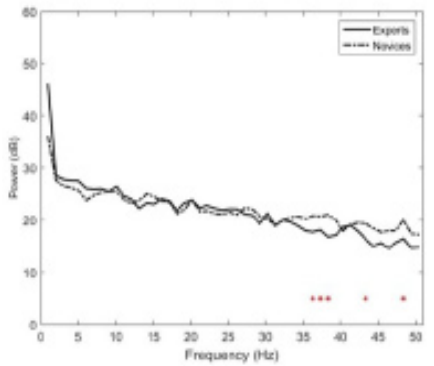

6c. Right temporal cluster

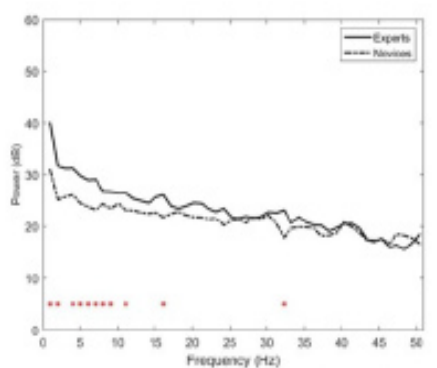

6d. Left lateral parietal cluster
Figure 6: Spectral power and Wilcoxon signed-rank test results for the productivity indicator during the idea generation task

The participants exhibited high brain activation in the prefrontal and frontal cortices when engaged in the idea evaluation task of the productivity indicator (Figure 7). The scalp topographies and 3D dipole plots presented in Figures $7 \mathrm{a}$ and $7 \mathrm{~b}$ reveal that these brain activations can be divided into two major component clusters, namely the right prefrontal and left frontal cortices. The correlation between these two clusters was relatively high. Our results indicated that two significant power differences (EDs > NDs) in the right prefrontal cluster were observed in 
the gamma band at $34 \mathrm{~Hz}(\mathrm{p}=0.021)$ and the theta band at $7 \mathrm{~Hz}$ ( $p=0.041$ ) (Figure 8a). Additionally, significant power differences were observed at most frequencies in the left frontal cluster (Figure $8 b)$, where the largest differences appeared (EDs $>$ NDs) in the alpha band at $11 \mathrm{~Hz}(p=0.001)$ and $10 \mathrm{~Hz}(p=0.001)$.
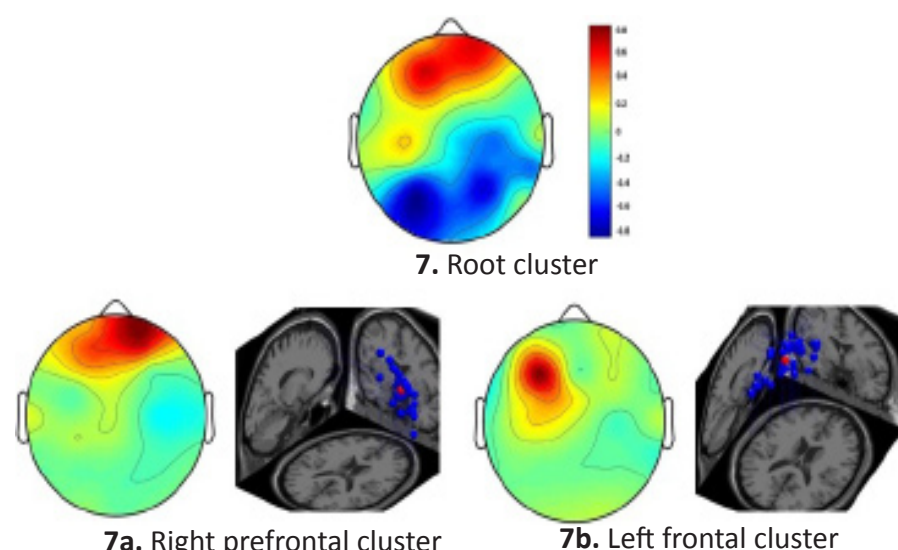

7b. Left frontal cluster

Figure 7: Scalp topographies and 3D dipole plots for the productivity indicator during the idea evaluation task

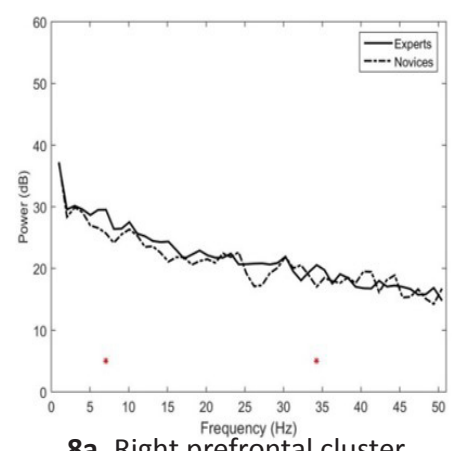

8a. Right prefrontal cluster

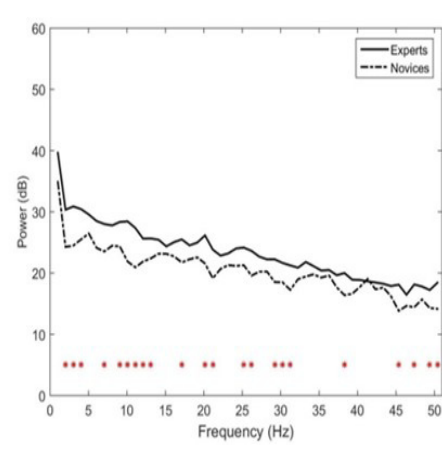

8b. Left frontal cluster
Figure 8: Spectral power and Wilcoxon signed-rank test results for the productivity indicator during the idea evaluation task

\section{Discussion}

\section{Novelty indicator for the idea generation task}

The results indicated that the prefrontal and bilateral frontotemporal regions were activated during the experimental task. In particular, the activations in the right prefrontal, left temporal, right temporal, and left frontal cortices were evident. The major functions of the right temporal cortex are nonverbal memory and communication [28], whereas the left frontal cortex is in charge of analogical reasoning. These capabilities are critical for making inferences and adapting to novelty [29]. The high correlation between the right temporal and left frontal cortices appears to enable designers to apply nonverbal memory for analogical reasoning to make novel inferences and generate ideas.

The right prefrontal cortex is in charge of empathy and regulating emotional responses [30,31], as well as visual association and insightful problem-finding processes [32], thereby implying that emotional regulation and empathy facilitate creative association and performance in designers. The left temporal cortex controls low-level perception, extending to verbal memory and comprehension. Optimum activity in the left temporal cortex enhances mood stability $[33,34]$. The coactivation of the right prefrontal and left temporal cortices appears to assist designers in regulating emotional responses, which may facilitate the efficient augmentation of their cognitive capacities in recognising design novelty.

As depicted in Figures $2 a-2 d$, the spectral powers of the EDs and NDs for the novelty indicator during the idea generation task were mostly similar. Although no significant power differences were observed in the right prefrontal cluster, one significant power difference (EDs > NDs) was observed in the beta band in the left temporal cluster, implying that EDs are more competent at consciously regulating emotions for novel ideation than are NDs. Additionally, the power differences (EDs > NDs) in the right temporal cluster and those (NDs > EDs) in the left frontal cluster appeared in the beta and gamma bands, implying that the EDs are more competent at using nonverbal memory, while the NDs are more competent at practicing analogical reasoning for novel ideation. Overall, these results implied that by optimising the designers' capability to regulate emotions and use nonverbal memory with abstract visual stimuli, NDs can enhance their ability to generate novel ideas.

\section{Novelty indicator for the idea evaluation task}

The EEG results indicated that the right frontal and temporal regions were activated during the idea evaluation task. In particular, the activations of the left temporal, right-biased middle frontal, right lateral frontal, and right temporal cortices were noticeable. As previously stated, the left temporal cortex manages verbal memory and comprehension and its activation can improve mood stability $[33,34]$. The right-biased middle frontal region was closely associated with the ACC, which controls mnemonic conflict detection and resolution by monitoring differential familiarity [35]. The negative correlation between these two regions (Table 2 ) was minor slight but significant, implying that for the evaluation of idea novelty, the function of conflict management can be used as a lever to facilitate emotional stability, and vice versa.

The right frontal cortex is critical for the various types of divergent semantic processing involved in human creativity [36] and the right temporal cortex is crucial for nonverbal memory. The negative correlation between these two regions (Table 2 ) implies that while a designer engages in an evaluation task involving idea novelty, depressing nonverbal communication may facilitate divergent semantic processing, and vice versa. In addition, the right-biased middle frontal region was positively correlated to the right temporal cortex, implying that activating nonverbal communication could ease mnemonic conflict management. Synthesising these results yields the suggestion that the right temporal cortex plays a critical role in moderating the functions handled by the ACC in the idea novelty evaluation task.

As depicted in Figures 4a-4d, power differences (NDs > EDs) in the left temporal, right-biased middle frontal, and right temporal clusters were evident and the differences appeared in distributed frequencies. These results revealed that during the idea evaluation task, complicated interactions involving emotional stability, conflict management, and nonverbal memory were more active in the NDs than in the EDs. These results alone cannot definitively conclude that NDs are more competent than EDs in managing these intertwined interactions or that EDs expend less energy than do NDs in reacting to interactions between different brain regions. However, the critical role of emotion and the sophisticated relationships between regions close to the ACC during idea evaluation warrant further inquiry. 


\section{Productivity indicator for the idea generation task}

During the idea generation task for the productivity indicator, noticeable activations in the right-biased middle frontoparietal region were detected. In particular, the activations in the right medial parietal, middle frontal, right temporal, and left lateral parietal cortices were manifest. The right medial parietal cortex is a crucial part of the PCC that serves to reinstate familiar contextual information from memory [37]. The PCC exhibits a wide range of connectivity with the DMN, the activation of which is related to a high level of self-generated thought [11,17]. In addition, the middle frontal cortex is associated with the ACC, which is involved in managing conflict detection and resolution [35]. The coactivations of these two regions (Table 3 ) indicated that simultaneously managing mnemonic conflicts and focusing on internally directed thoughts may facilitate the fluency of idea generation in designers.

Whereas the right temporal cortex is associated with nonverbal memory and information processing, the left parietal cortex promotes the flexible integration of prior knowledge to construct new ideas [38]. Thus, these results implied that designers adaptably integrate nonverbal memory to productively construct design ideas. The high correlation between the right medial parietal and left lateral parietal cortices (Table 3 ) implies that the fluency of idea generation mainly relies on the flexible integration of self-generated thoughts, including autobiographical memory retrieval, episodic future thinking, and mind wandering based on personal experiences [16,39]. Moreover, the high correlation between the middle frontal and left lateral parietal cortices (Table 3 ) implies that the fluency of design ideation depends on how efficiently the ACC detects and resolves mnemonic conflicts.

During this task, the theta and alpha power of the EDs was generally higher than that of the NDs in all four component clusters. An increase in alpha power in the right parietal cluster is an indicator of the depth or elaborateness of an ongoing process of mental imagination [40], through which EDs can outperform NDs with respect to the fluency of design ideation. Previous studies have reported that greater theta and alpha power reflects higher internal processing demand $[18,41]$. In addition, our results suggest that the EDs devoted more energy than the NDs to flexibly integrating past knowledge for idea construction (left lateral parietal cortex). Furthermore, the activation of the right temporal cortex in the NDs was higher than that of the EDs in the gamma band, implying that NDs consciously use their nonverbal memory to facilitate the productivity of design ideation.

\section{Productivity indicator for the idea evaluation task}

During the idea evaluation task for the productivity indicator, noticeable activations were detected in the prefrontal and frontal regions. In particular, the activations of the right prefrontal and left frontal cortices were prominent. As previously stated, the right prefrontal cortex plays a fundamental role in emotional regulation [30,31]. The left frontal cortex is in charge of analogical reasoning and concept formation and the nearby ACC maintains cognitive control for detecting and resolving conflicts. These results implied that the designers in this study perceived the idea evaluation process to entail conflict detection, monitoring, and resolution, as well as the evaluation of idea productivity to improve outcomes through emotional regulation-possibly to facilitate assessment efficiency. The high correlation between the right prefrontal and left frontal corti- ces (Table 4) represents the necessary coactivation of these two regions in idea productivity evaluation.

As depicted in Figures $8 a$ and $8 b$, the spectral power of the EDs was generally higher than that of the NDs for the novelty indicator during the idea generation task. Two significant power differences were observed in the theta and gamma bands in the right prefrontal cluster, implying that EDs are more competent at regulating emotions for ideation productivity than are NDs. In addition, the power differences (EDs > NDs) in the left frontal cluster appeared at most frequencies, implying that EDs are more competent at analogical reasoning and concept formation than are NDs. Taken together, these results implied that by optimising their capability for emotional regulation and analogical reasoning, NDs' can enhance their ability to evaluate idea productivity.

\section{Research limitations}

This study had several limitations. First, EEG enabled the waveform elicited by certain tasks to be determined through averaging several trials. However, its low spatial resolution on the scalp hinders the tracing of the exact point in the brain where an activity originates, particularly regarding those in the medial or orbital regions related to human emotions. Second, the experimental stimuli used in the present study were limited to the paintings of Picasso. Additional visual representations and other forms of stimuli (e.g., drawings, photos, videos, music, and 3D objects) could be employed. Third, neuroscientific studies have typically investigated simple cognitive processes by using repeatable tasks. However, designer imagination and design thinking are multifaceted mental activities, and thus creating reliable and valid experimental processes and settings in which the research objective can be fulfilled is challenging. Finally, we cannot completely exclude the possibility that the participants had multiple and mixed ideas, which may have hindered the interpretation of idea generation and evaluation.

\section{Closing remarks/Conclusion}

Based on the results, several practical implications can be derived. (i) By optimising their capability to regulate emotions and use nonverbal memory, designers can enhance their ability to generate novel ideas. (ii) By leveraging their capability for emotional management, conflict detection, and nonverbal memory, designers can facilitate their evaluation of novel ideas. (iii) By augmenting their capability for mnemonic conflict management and autobiographical memory retrieval, particularly in terms of theta and alpha power, designers can improve their productivity regarding idea generation. (iv) By enriching their capability for emotional management and analogical reasoning, designers can increase their assessment efficiency as regards idea evaluation. EDs are expected to be responsible for conceptualisation, creative development, and final design execution. The aforementioned practical implications provide insightful suggestions for ideation enhancement that can be applied in in-service training and formal design education.

Experience enables designers to overcome diverse obstacles during their professional lives. As demonstrated in the present study, neuroscience provides instruments and methods that can be applied to study design competencies. The results of this study provide new evidence to facilitate future studies of designer imagination and new insights to differentiate the cognitive processes of visual designers of various experience levels. We hope that these ideas will stimulate future research, the de- 
velopment of novel paradigms, and critical scientific exchange between the research communities involved in understanding different aspects of designer imagination. Through this discussion, we hope that building blocks and essential frameworks will be established to serve as guides to the prolific world of the human imagination.

\section{References}

1. Chang CC, Wang JH, Liang CT, Liang C. Curvilinear effects of openness and agreeableness on the imaginative capability of student designers. Thinking Skills and Creativity. 2014; 14: 68-75.

2. Liu E, Noppe-Brandon, S. Imagination first: Unlocking the power of possibilities. San Francisco, CA: Jossey-Bass. 2009.

3. Liang C, Chia TL. Reliability, validity, and factor structure of the imaginative capability scale. Creativity Research Journal. 2014; 26: 106-114.

4. Mayseless N, Aharon-Peretz J, Shamay-Tsoory S. Unleashing creativity: The role of left temporoparietal regions in evaluating and inhibiting the generation of creative ideas. Neuropsychologia. 2014; 64: 157-168.

5. Yao SN, Lin $\mathrm{CT}$, King JT, Liu YC, Liang C. Learning in the visual association of novice and expert designers. Cognitive Systems Research. 2017; 43: 76-88.

6. Chevalier A, Ivory MY. Web site designs: Influences of designer's expertise and design constraints. International Journal of $\mathrm{Hu}$ man-Computer Studies. 2003; 58: 57-87.

7. Julier G, Moor L. Design and creativity: Policy, management and practice. New York, NY: Bloomsbury Academic. 2009.

8. Lloyd P, Scott P. Discovering the design problem. Design Studies. 1994; 15: 125-140.

9. Cross N. Expertise in design: An overview. Design Studies. 2004; $25 ; 427-441$

10. Dorst K, Cross N. Creativity in the design process: Co-evolution of problem-solution. Design Studies. 2001; 22: 425-437.

11. Liang C, Lin CT, Yao SN, Chang WS, Liu YC, et al. Visual attention and association: An electroencephalography study in expert designers. Design Studies. 2017; 48. 76-95.

12. Ball LJ, Ormerod TC, Morley NJ. Spontaneous analogising in engineering design: A comparative analysis of experts and novices. Design Studies. 2004; 25: 495-508.

13. Kavakli M, Gero JS. The structure of concurrent cognitive actions: A case study on novice and expert designers. Design Studies. $2012 ; 23: 25-40$.

14. Ericsson KA. Protocol analysis and expert thought: Concurrent verbalizations of thinking during experts' performance on representative tasks. In K. Ericsson, N. Charness, P. J. Feltovich, \& R. R. Hoffman (Eds.), Cambridge handbook of expertise and expert performance . Cambridge, UK: Cambridge University Press. 2006; 223-242.

15. Goldschmidt G. Ubiquitous serendipity: Potential visual design stimuli are everywhere. In J. S. Gero (Ed.) Studying visual and spatial reasoning for design creativity. Netherlands: Springer. 2015; 205-214.

16. Buckner RL, Andrews-Hanna JR, Schacter DL. The brain's default network: Anatomy, function and relevance to disease. Annals of the New York Academy of Sciences. 2008; 1124: 1-38.

17. Schacter DL, Addis DR, Hassabis D, Martin VC, Spreng RN. The future of memory: Remembering, imagining, and the brain.
Neuron. 2012; 76: 677-694

18. Fink A, Neubauer AC. EEG alpha oscillations during the performance of verbal creativity tasks: Differential effects of sex and verbal intelligence. International Journal of Psychophysiology. 2006; 62: 46-53.

19. Lustenberger C, Boyle MR, Foulser AA, Mellin JM, Fröhlich F. Functional role of frontal alpha oscillations in creativity. Cortex. 2015; 67: 74-82.

20. Cila N, Hekkert, P, Visch V. Source selection in product metaphor generation: The effects of salience and relatedness. International Journal of Design. 2014; 8: 15-28.

21. Goldschmidt G, Smolkov M. Variances in the impact of visual stimuli on design problem solving performance. Design Studies. 2016; 27: 549-569.

22. Edens KM, Potter EF. Promoting conceptual understanding through pictorial representation. Studies in Art Education. 2001; 42; 214-233.

23. Wang YK, June TP, Lin CT. EEG-based attention tracking during distracted driving. IEEE Transactions on Neural Systems and Rehabilitation Engineering. 2015; 23: 1085-1094.

24. Onton J, Westerfield M, Townsend J, Makeig S. Imaging human EEG dynamics using independnt component analysis. Neuroscience and Biobehavioral Reviews. 2006; 30: 808-822.

25. Cantero JL, Atienza M, Gómez CM, Salas RM. Spectral structure and brain mapping of human alpha activities in different arousal states. Neuropsychobiology. 1999; 39: 110-116.

26. Grützner C, Wibral M, Sun M, Rivolta D, Singer Wet al. Deficits in high- (>60 Hz) gamma-band oscillations during visual processing in schizophrenia. Frontiers in Human Neuroscience. 2013.

27. Tang $\mathrm{Y}, \mathrm{Chorlian} \mathrm{DB}$, Rangaswamy $\mathrm{M}$, Porjesz B, Bauer L, et al. Genetic influences on bipolar EEG power spectra. International Journal of Psychophysiology. 2007; 65; 2-9.

28. Wisniewski I, Wendling AS, Manning L, Steinhoff BJ. Visuo-spatial memory tests in right temporal lobe epilepsy foci: Clinical validity. Epilepsy \& Behavior. 2012; 23: 254-260.

29. Fine EM, Delis DC, Dean D, Beckman V, Miller BL, et al. Left frontal lobe contributions to concept formation: A quantitative MRI study of D-KEFS sorting test performance. Journal of Clinical and Experimental Neuropsychology. 2009; 31: 624-631.

30. Gallup GGJ, Platek SM. Cognitive empathy presupposes selfawareness: Evidence from phylogeny, ontogeny, neuropsychology and mental illness. Behavioral and Brain Sciences. 2002; 25: 36-37.

31. Ochsner KN, Silvers JA, Buhle JT. Functional imaging studies of emotion regulation: A synthetic review and evolving model of the cognitive control of emotion. Annals of the New York Academy of Sciences. 2012; 1251: E1-24.

32. Liu YC, Yang YH, Liang C. How do creativity levels and stimulus types matter? A preliminary investigation of designer visual association. Journal of Neurology \& Neuroscience. 2017; 8: 1-13.

33. Amen DG. Healing the hardware of the soul: How making the brain-soul connection can optimize your life, love, and spiritual growth. New York, NY: Free Press. 2002.

34. Crinion JT, Lambon-Ralph MA, Warburton EA, Howard D, Wise, RJS. Temporal lobe regions engaged during normal speech comprehension. Brain. 2013; 126; 1193-1201.

35. Bunge SA, Burrows B, Wagner AD. Prefrontal and hippocampal contributions to visual associative recognition: Interactions be- 
tween cognitive control and episodic retrieval. Brain and Cognition. 2004; 56: 141-152.

36. Howard-Jonesa, PA, Blakemore SJ, Samuela EA, Summers IR, Claxton G. Semantic divergence and creative story generation: An fMRI investigation. Cognitive Brain Research. 2005; 25: 240250.

37. Szpunar KK, Chan JC, McDermott KB. Contextual processing in episodic future thought. Cerebral Cortex. 2009; 19: 1539-1548.

38. Benedek M, Jauk E, Fink A, Koschutnig K, Reishofer Get al. To create or to recall? Neural mechanisms underlying the generation of creative new ideas. Neurolmage. 2014; 88: 125-133.

39. Beaty RE, Benedek M, Wilkins RW, Jauk E, Fink Aet al. Creativity and the default network: A functional connectivity analysis of the creative brain at rest. Neuropsychologia. 2014; 64: 92-98.

40. Von Stein A, Sarnthein J. Different frequencies for different scales of cortical integration: From local gamma to long range alpha/ theta synchronization. International Journal of Psychophysiology. 2000; 38: 301-313.

41. Schwab D, Benedek M, Papousek I, Weiss EM, Fink A. The timecourse of EEG alpha power changes in creative ideation. Frontiers in Human Neuroscience. 2014. 